\title{
Ryanodine Receptor 2 Plays a Critical Role in Spinal Cord Injury via Induction of Oxidative Stress
}

\author{
Bo Liao Yong Zhang Honghui Sun Baoan Ma Jixian Qian \\ Department of Orthopaedics, Tangdu Hospital, Fourth Military Medical University, Xi'an, China
}

\section{Key Words}

Spinal cord injury • Ryanodine receptor 2 - Inflammation • Oxidative stress $\bullet$ NADPH oxidase 2

\begin{abstract}
Background/Aims: Spinal cord injury (SCI) is a severe health problem worldwide. Ryanodine receptors (RyRs) are a class of intracellular calcium channels in various excitable tissues such as muscles and nervous tissues. The current study was designed to investigate the possible role of RyR2 upregulation in SCI and to elucidate the possible molecular mechanisms. Methods: Rats were injected with LVshRNAi- RyR2 and then exposed to spinal cord contusion injury. Results: The results showed that knockdown of RyR2 significantly promoted the recovery of structural and functional injury in spinal cord, as evidenced by reduction of lesion volume and increase of Basso, Beattie and Bresnahan (BBB) and combined behavioral score (CBS) scores. Knockdown of RyR2 inhibited the increase of proinflammatory cytokines, including IL-1 $\beta$ and TNF $\alpha$. Moreover, downregulation of RyR2 increased oxygen consumption rate and decreased the expression of glucose-regulated protein 78 (GRP78), activating transcription factor 3 (ATF3) and ATF6, indicating the improvement of mitochondrial dysfunction and endoplasmic reticulum stress after SCI. Furthermore, silence of RyR2 reduced oxidative stress, as reflected by decrease of TBARS and GSSG content and increase of GSH level. The expression of NADPH oxidase 2 (NOX2), NOX4 and p66 $6^{\text {shc }}$ were increased in SCI rats. Knockdown of RyR2 significantly decreased NOX2 expression, but had no evident effect on NOX4 and p66 shc expression. These results indicated NOX2 may be involved in RyR2-induced ROS generation which mediated contusion-induced spinal cord injury. Conclusion: The data provide novel insights into the mechanism of RyR2-mediated injury and the potential therapeutic targets for injury in spinal cord.




\section{Introduction}

Spinal cord injury (SCI) is a severe health problem worldwide [1]. SCI could severely impair neural structure and function, resulting in reduction of quality of life in patients [25]. SCI is a biphasic injury process including primary and secondary injury. The primary injury, caused by initial physical impact, is characterized by acute bleeding and ischemia. After primary injury, secondary injury can be triggered by several mechanisms, including inflammation, oxidative stress, apoptosis and degeneration of neurons [6, 7]. To date, loss of neuron resulted by primary injury could not be reversed. Therefore, most of the attention has been paid on molecular mechanism and therapeutic strategy of the secondary injury. However, the molecular mechanism of secondary damage after SCI is still largely unknown.

Ryanodine receptors (RyRs) are a class of intracellular calcium channels in various excitable tissues such as muscles and nervous tissues [8-10]. RyRs consist of three major isoforms (RyR1, 2, and 3), which are involved in calcium release from different intracellular organelles via differential signaling pathways [11]. RyRs mediate the release of calcium ions from the sarcoplasmic reticulum and endoplasmic reticulum [12]. Calcium influx and elevation of intracellular free calcium activate degenerative enzymes, causing cell injury and death after trauma. Blockade of calcium release through RyRs confers significant improvement of SCI [13]. Moreover, RyR2 expression in astrocyte and axons is markedly increased after hypoxic injury of spinal cord dorsal column [14]. However, mechanisms underlying RyR2-associated development of SCI are not clear.

The current study was designed to investigate role of RyR2 upregulation in SCI and to elucidate the possible molecular mechanisms. We found that knockdown of RyR2 decreased NADPH oxidase 2 (NOX2) expression, reduced oxidative stress, improved mitochondrial dysfunction and endoplasmic reticulum (ER) stress, prohibited inflammation, resulting improved recovery of SCI. We suggest that RyR2 play an important role in SCI through regulation of redox state and thus mitochondrial and ER function and inflammation.

\section{Materials and Methods}

\section{Chemicals and reagents}

$\beta$-actin and RyR2 antibodies were obtained from Santa Cruz. GRP78, ATF3 and ATF6 antibodies were obtained from Cell Signaling Technology. Most of the other reagents were obtained from Sigma.

\section{Animal treatment}

Adult male Sprang-Dawley rats (200-250 g) were purchased from the Experimental Animals Center of Fourth Military Medical University. All animal experiments were conducted in accordance with the National Institute of Health Guide on the Care and Use of Laboratory Animals and were approved by the Laboratory Animal Users Committee at Fourth Military Medical University. All animals were housed in individual cages in a temperature and light \pm dark cycle controlled environment with free access to food and water. A midthoracic (T9) laminectomy was conducted in rats and LVshRNAi-RyR2 or LV-Ctrl was injected $\left(3 \times 10^{7}\right.$ transducing units in $1 \mu \mathrm{l}$ ) at both the rostral and caudal ends of the laminectomy site using a Hamilton syringe (Hamilton, Reno, NV, USA) with a bevelled needle as previously reported [15]. During the injection, the syringe was inserted to 1-2 mm beneath the dorsal surface of the spinal cord, and $2 \mu \mathrm{l}$ of $\mathrm{LV}$ was injected at a rate of $1 \mu \mathrm{l}$ per 15 min by a Legato 130 Syringe Pump (KD Scientific, Holliston, MA, USA). Rats in the sham group received a laminectomy without injection $(n=10)$. After the injection, rats were monitored for 1 week to ensure viral incorporation and efficiency of knockdown of target gene. 2 days after injection, functional observation was conducted using locomotor testing to evaluate whether there was evident damage caused by the operation. After 1 week recovery, the laminectomy site was exposed and a moderate spinal cord contusion injury was established by dropping a $10 \mathrm{~g}$ weight from a height of $5 \mathrm{~cm}$ onto an NYU impactor positioned on the exposed site [16-18]. Rats were sacrificed at 1 day ( $n=8 /$ group), 3 days ( $n=8 /$ group), 7 days ( $\mathrm{n}=8$ /group), 14 days ( $\mathrm{n}=8$ /group), 21 days ( $\mathrm{n}=8$ /group) and 28 days ( $\mathrm{n}=8$ /group) after injury. After the treatment, a 5-mm spinal cord segment, $2.5 \mathrm{~mm}$ rostral and $2.5 \mathrm{~mm}$ caudal to the lesion epicenter, was collected and used for the following detections, respectively.

\section{KARGER}




\section{Cellular Physiology Cell Physiol Biochem 2016;38:1129-1137

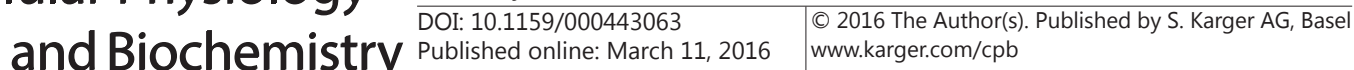

Liao et al.: Critical Role of Ryanodine Receptor 2 in Spinal Cord Injury

Assessment of motor function

Locomotor function of hind limb was assessed using the Basso, Beattie and Bresnahan (BBB) rating scale [19]. A combined behavioral score (CBS) was also conducted to evaluate neurological functional deficits as previously described $[20,21]$. Two independent and well-trained investigators blinded to treatment conducted the experiments and scored the locomotor function and neurological function at 7, 14, 21 and 28 days after the injury [22].

Determination of proinflammatory cytokines

The spinal cord tissues were homogenated and the content of IL-1 $\beta$ and TNF $\alpha$ in spinal cord tissue homogenates were measured using ELISA kits according to the manufacturers' instructions (Abcam).

\section{Protein extraction and western blot}

Total protein was extracted using RIPA lysis buffer according to the manufacturer's protocols. Protein concentrations in supernatants were measured using a Bradford protein assay kit (Pierce, USA). About $20 \mu \mathrm{g}$ of protein lysis was separated using SDS-PAGE. Then, protein was transferred to PVDF membranes (Millipore, MA, USA), which were then blocked with $5 \%$ non-fat milk. Membranes were incubated with primary antibodies overnight at $4{ }^{\circ} \mathrm{C}$. After washing, the membrane was incubated with horseradish peroxidase-conjugated secondary antibodies (Pierce, USA) at $37^{\circ} \mathrm{C}$ for $1 \mathrm{~h}$. Protein bands were visualized with the ECL and captured using BIORAD Imaging Systems (BIORAD, CA, USA).

Real-time PCR

Total RNA was extracted using the Trizol method, according to the manufacturer's instructions (Invitrogen, USA). After reverse transcription, quantitative real-time PCR (qRT-PCR) was analyzed in a BIORAD CFX96 Real-Time System, using SYBR Premix Ex TaqTM II (Takara, Japan). The expression of target genes was normalized to $\beta$-actin and the gene expression was comparatively analyzed using the $2^{-\Delta \Delta \mathrm{Ct}}$ method.

\section{Oxygen consumption}

The spinal cord tissues were homogenated and oxygen consumption was recorded for 10 min using a Clark Oxygen Electrode. Oxygen consumption rate was calculated and expressed as percentage of remaining oxygen in the reaction mixture.

Assessment of oxidative stress

The spinal cord tissues were homogenated and the content of TBA-reactive substances (TBARS), glutathione (GSH) and oxidized form of GSH (GSSG) levels were measured using commercial kits according to the manufacturers' instructions (Nanjing Jiancheng, China).

\section{Statistical analysis}

The results were presented as means \pm SEM. Results were analyzed using GraphPad Software. For two independent group comparisons (lesion volume, BBB and CBS scores), student t-test was conducted. The statistical significance of differences between three groups (proinflammatory cytokines, oxygen consumption rate, and oxidative stress-related parameters) was analyzed via one-way analysis of variance followed by a Dunnett's t-test for multiple comparisons. A P value $<0.05$ was considered to be statistically significant.

\section{Results}

Knockdown of RyR2 facilitates the recovery after SCI

To investigate the role of RyR2 in SCI, rats were injected with lentivirus-mediated shRNAi before the injury operation. In Fig. 1, we showed that 1, 14 and 28 days after the injury, RyR2 expression was significantly increased, compared with that of Sham group. In LVshRNAi-RyR2 group, expression of RyR2 in spinal cord was notably prohibited (Fig. 1). The results indicated that LVshRNAi-mediated knockdown of RyR2 was significant. 
Fig. 1. Effect of knockdown of RyR2 on RyR2 expression during spinal cord recovery. At 1, 14, and 28 days post-injury, western blot was conducted to determine protein expression of RyR2 in spinal cord. $* \mathrm{P}<0.05$, compared with Sham group. $* * \mathrm{P}<0.05$, compared with the LV-Ctrl group.

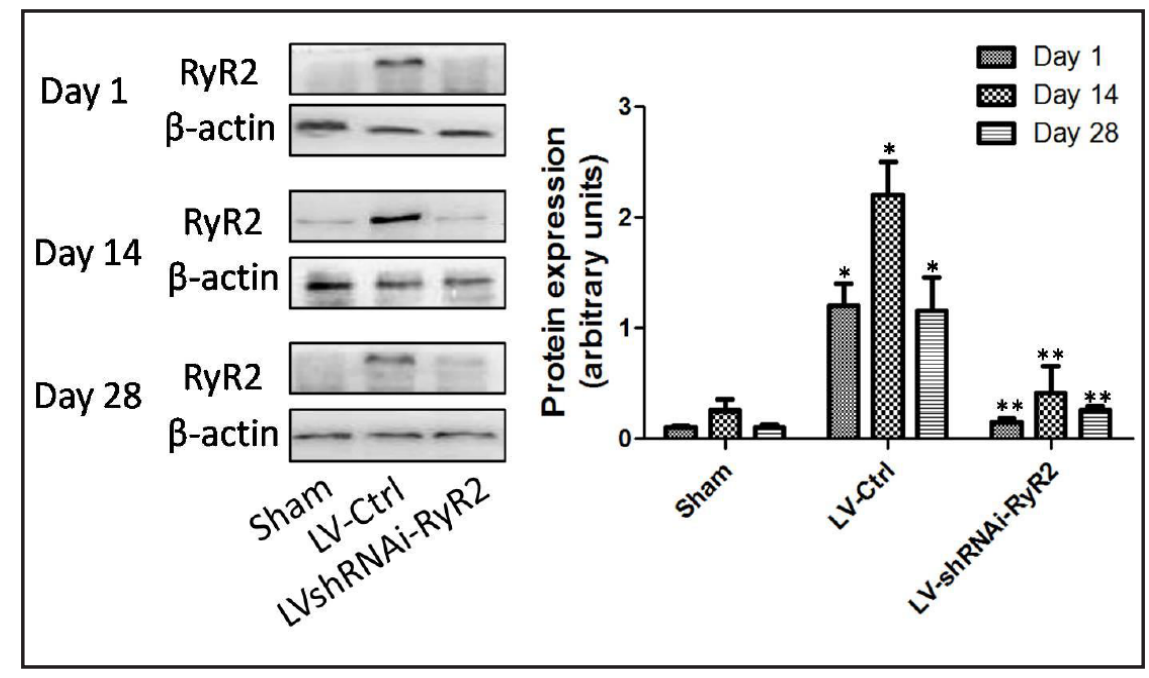

Fig. 2.Effect of knockdown of RyR2 on lesion volume and locomotor function recovery. (A) 28 days after the injury, stereological assessment was performed to assess lesion volume and quantitative analysis of lesion volume was showed. (B and C) At 7, 14, 21 and 28 days post-injury, the Basso-Beattie-Bresnahan (BBB) score (B) and combined behavioral score (CBS) (C) of rats were observed. $* \mathrm{P}<0.05, \quad$ compared with the LV-Ctrl group.

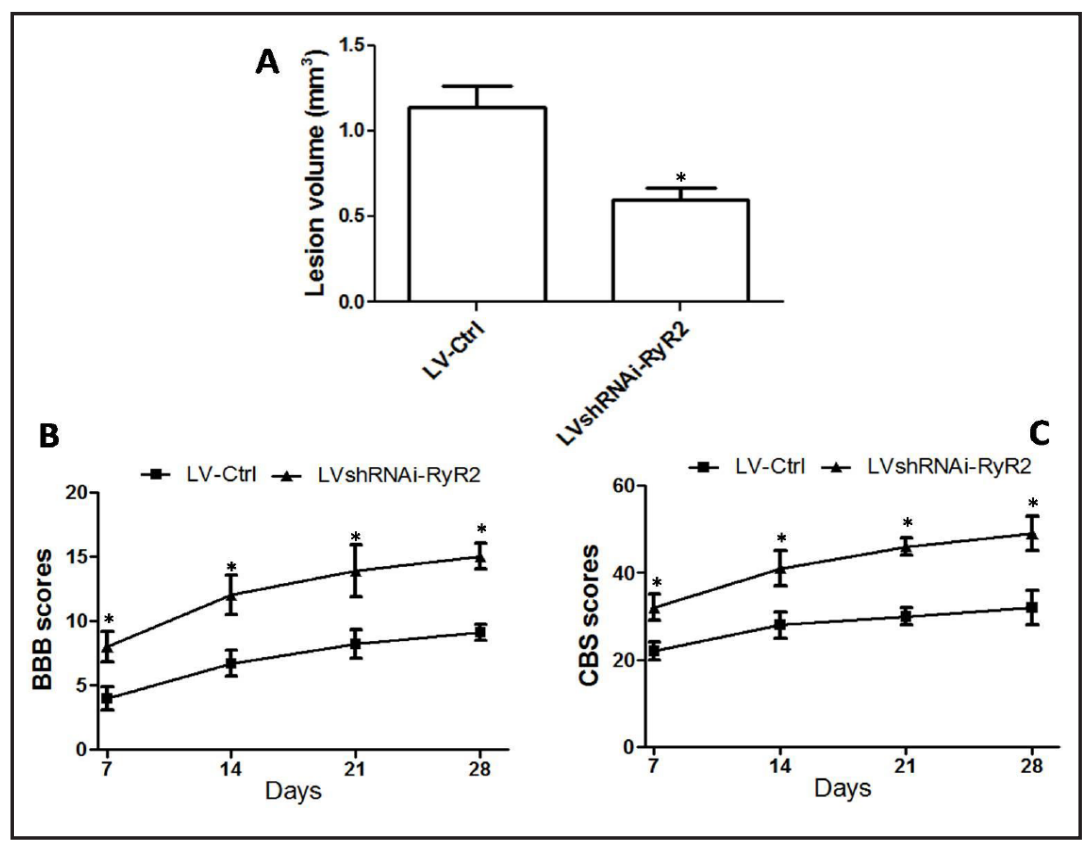

As shown in Fig. 2A, LVshRNAi-RyR2 treatment notably decreased the lesion volume of spinal cord in rats. In addition, the results of locomotor and neurological function showed that knockdown of RyR2 markedly increased the BBB (Fig. 2B) and CBS (Fig. 2C) scores, compared with LV-Ctrl groups. These results indicated that upregulation of RyR2 was involved in contusion-induced SCI and knockdown of RyR2 could facilitate the recovery after SCI.

Knockdown of RyR2 inhibits inflammation after SCI

To evaluate potential effects of RyR2 on inflammation after SCI, proinflammatory cytokines were determined. As reflected in Fig. 3, IL-1 $\beta$ and TNF $\alpha$ levels after SCI were significantly higher than that of Sham group and knockdown of RyR2 remarkably inhibited the increase of IL-1 $\beta$ and TNF $\alpha$ levels. The results demonstrated that upregulation of RyR2 was involved in SCI-associated inflammation. 

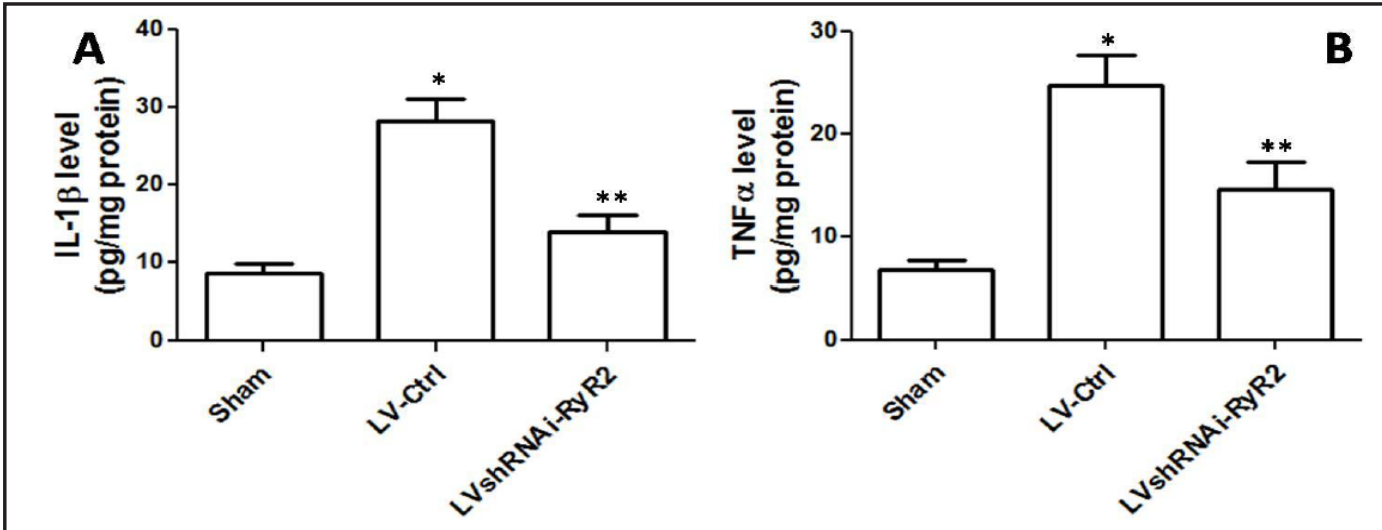

Fig. 3. Effect of knockdown of RyR2 on inflammation in spinal cord. 28 days after the injury, the spinal cord tissues were homogenated and the content of IL-1 $\beta$ (A) and TNF $\alpha(B)$ in spinal cord tissue homogenates were measured using ELISA kits. ${ }^{*} \mathrm{P}<0.05$, compared with Sham group. ${ }^{* *} \mathrm{P}<0.05$, compared with the LV-Ctrl group.

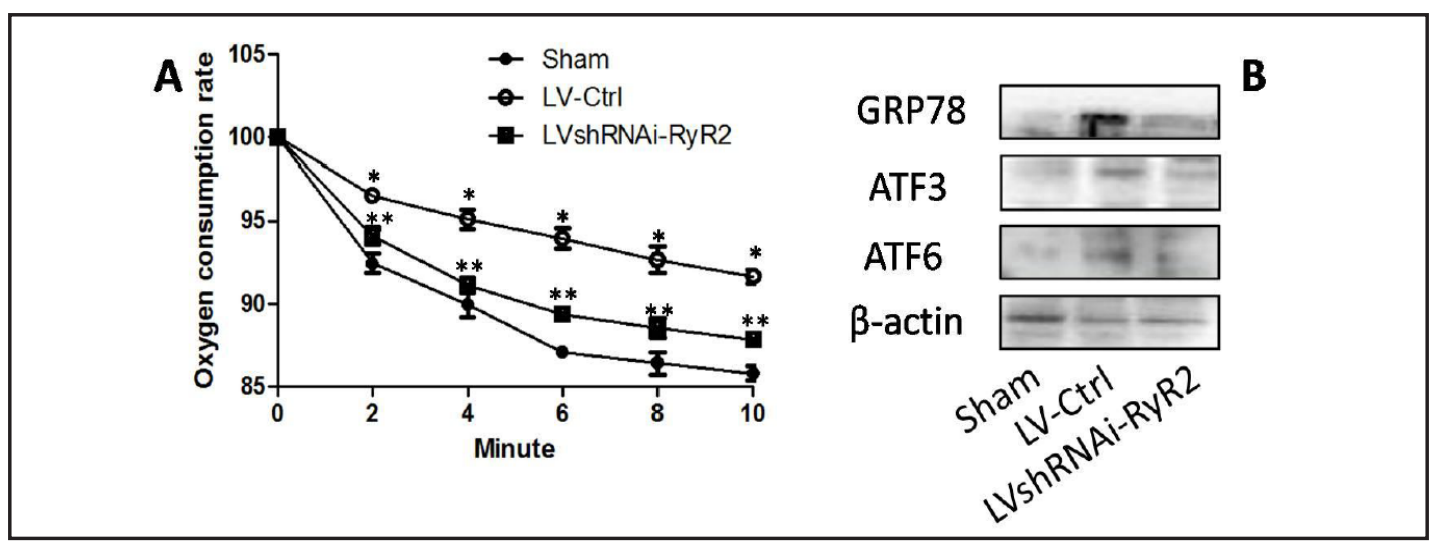

Fig. 4. Effect of knockdown of RyR2 on mitochondrial and ER function in spinal cord. 28 days after the injury, the spinal cord tissues were collected. (A) Oxygen consumption rate of spinal cord tissue was evaluated using an oxygen electrode. (B) Protein expression of GRP78, ATF3 and ATF6 in spinal cord tissue was determined by western blot. ${ }^{*} \mathrm{P}<0.05$, compared with Sham group. ${ }^{* *} \mathrm{P}<0.05$, compared with the LV-Ctrl group.

\section{Knockdown of RyR2 improves mitochondrial dysfunction and ER stress after SCI}

Next, we examine the possible effect of knockdown of RyR2 on mitochondrial and ER function. In Fig. 4A, we showed that contusion injury significantly decreased the oxygen consumption rate, compared with that of Sham group. In contrast, knockdown of RyR2 markedly inhibited the decrease of oxygen consumption rate in LV-Ctrl group. The results implicated that contusion-associated upregulation of RyR2 mediated mitochondrial dysfunction and knockdown of RyR2 prevented mitochondrial dysfunction. In addition, ER function was evaluated by the detection of sensitive markers. In Fig. 4B, we showed that expression of glucose-regulated protein 78 (GRP78), activating transcription factor 3 (ATF3) and ATF6 were significantly increased in rats with SCI, compared with that in Sham group. In LVshRNAi-RyR2 group, the increase of GRP78, ATF3 and ATF6 expression was significantly prohibited (Fig. 4C). The data demonstrated that upregulation of RyR2 may be involved in contusion-induced ER stress.

Knockdown of RyR2 reduces oxidative stress after SCI

As shown in Fig. 5A, TBARS content was significantly increased after SCI, compared with that of Sham group. Knockdown of RyR2 markedly inhibited the increase of TBARS (Fig. 5A). 


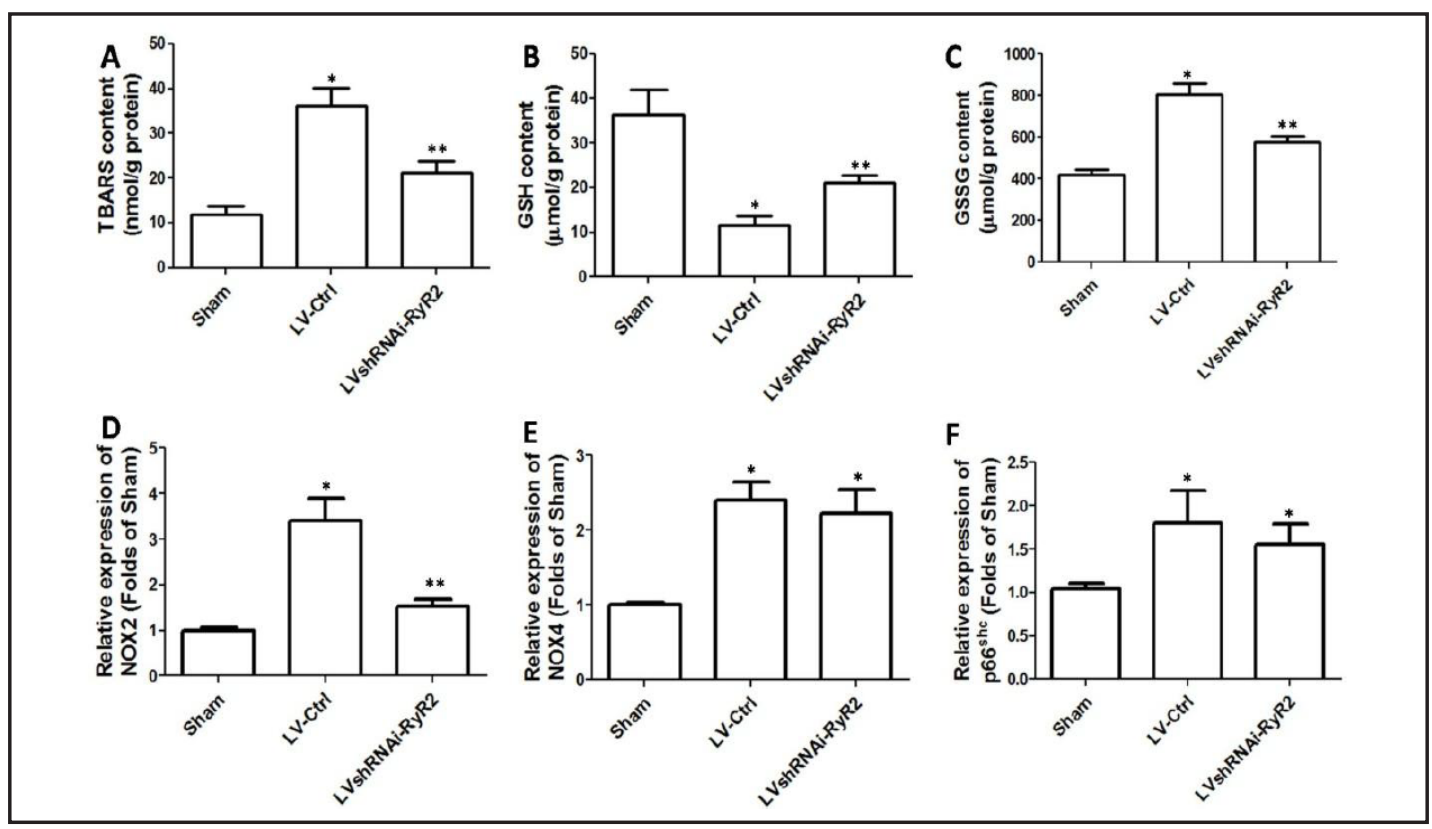

Fig. 5. Effect of knockdown of RyR2 on oxidative stress in spinal cord. 28 days after the injury, the spinal cord tissues were homogenated and the content of TBARS (A), GSH (B) and GSSG (C) levels were determined by commercial assay kits. mRNA expression of NOX2, NOX4 and P66 $6^{\text {shc }}$ was measured by real-time PCR. ${ }^{*} \mathrm{P}<0.05$, compared with Sham group. ${ }^{* *} \mathrm{P}<0.05$, compared with the LV-Ctrl group.

Moreover, GSH level was markedly reduced after SCI, which was inhibited by knockdown of RyR2 (Fig. 5B). The alteration of GSSG was opposite to that of GSH (Fig. 5C). The results indicated that contusion resulted in significant oxidative stress which may contribute to SCI and upregulation of RyR2 was involved in contusion-associated oxidative stress in spinal cord.

To explore the mechanism of RyR2-mediated oxidative stress, several key factors that are responsible for reactive oxygen species (ROS) generation were determined. As shown in Fig. 5, NADPH oxidase 2 (NOX2), NOX4 and p66 shc mRNA expression was measured by real-time PCR. mRNA expression of NOX2 in rats with SCI was significantly increased, compared with that of Sham group (Fig. 5A). Knockdown of RyR2 markedly inhibited the increase of NOX2 expression (Fig. 5D). In addition, NOX4 and p66 ${ }^{\text {shc }}$ mRNA expression were also increased by contusion in spinal cord (Fig. 5E and F). However, no significant effect of knockdown of RyR2 on NOX4 and p66 shc mRNA expression was observed (Fig. 5E and F). The results implicated that NOX2 may be involved in RyR2-mediated oxidative stress through ROS generation.

\section{Discussion}

Previous studies have shown that RyR2 expression is markedly increased after hypoxic injury of spinal cord dorsal column [14]. However, role of RyR2 in traumatic injury to spinal cord was largely unknown. In the present study, we knocked down the expression of RyR2 using LV construct and examined the effect of downregulation of RyR2 on SCI recovery. We found that knockdown of RyR2 significantly promoted the recovery of structural and functional injury in spinal cord, as evidenced by reduction of lesion volume and increase of BBB and CBS scores. Inflammation plays an important role in the injury of spinal cord after trauma and reduction of inflammation was considered to be an important intervention for SCI therapy $[23,24]$. Since downregulation of RyR2 prohibited the increase of proinflammatory 


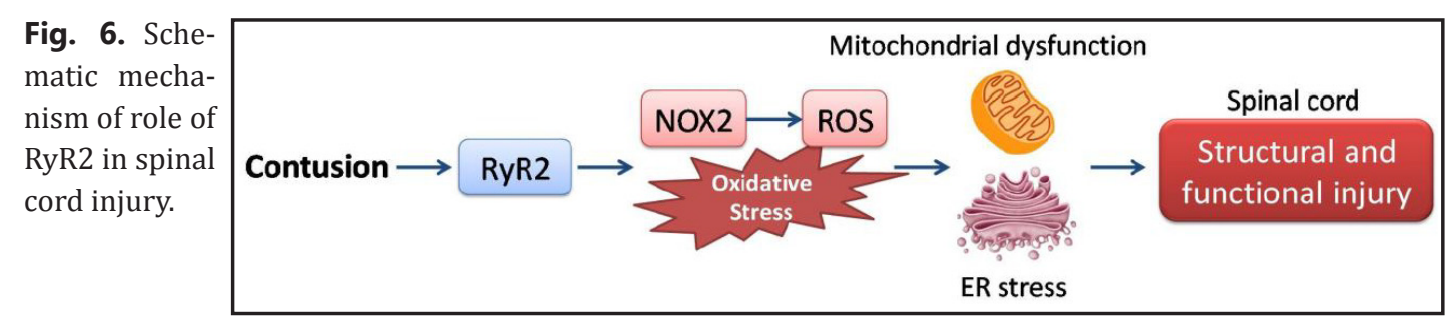

cytokines IL-1 $\beta$ and TNF $\alpha$, it was indicated that RyR2 was involved in the development of inflammation, which contributed to traumatic injury of spinal cord.

To further explore the mechanism of RyR2-associated reduction of inflammation and improvement of SCI, mitochondrial and ER function was evaluated. Mitochondrial dysfunction is an important pathophysiological process that is associated with various diseases. Especially, mitochondrial dysfunction is believed to be an important contributor to SCI [25-27]. In addition, ER stress participates in various types of damage after traumatic injury of spinal cord [28-30]. In the current study, we found that downregulation of RyR2 significantly ameliorated mitochondrial dysfunction and ER stress, as evidenced by increased oxygen consumption rate and decreased expression of GRP78, ATF3 and ATF6.

Under a variety of conditions, mitochondrial dysfunction and ER stress are closely associated with excessive ROS generation, also called oxidative stress. Oxidative stress was considered to be a central event in the development of SCI [31-34]. Activation of RyR2 contributes to excessive ROS generation which in turn results in mitochondrial dysfunction and ER stress [35-37]. In the present study, we further examined the effect of downregulation of RyR2 on oxidative stress. We showed that knockdown of RyR2 significantly reduced oxidative stress, as evidenced by decrease of TBARS and GSSG content and increase of GSH content. Furthermore, we also tested the possible source of ROS generation induced by RyR2. NOX2 and NOX4 are important ROS-generating enzymes mainly locating at membranes [38, 39]. $P 66^{\text {shc }}$ is a novel redox adaptor that could generate ROS in mitochondria [40]. We found that NOX2, NOX4 and $\mathrm{p} 66^{\text {shc }}$ were increased in SCI rats. It was previously found that inhibition of NOX2 reduced locomotor impairment, inflammation, and oxidative stress after SCI [41]. In the study, we found that knockdown of RyR2 significantly decreased NOX2 expression, but had no evident effect on NOX4 and p66 ${ }^{\text {shc }}$ expression. These results indicated NOX2 may be involved in RyR2-induced ROS generation which mediated contusion-induced SCI.

In conclusion, we show that knockdown of RyR2 decreased NOX2 expression, reduced oxidative stress, ameliorated mitochondrial dysfunction and ER stress, prohibited inflammation, resulting in improved recovery of SCI. Given the special role of RyRs in mediating the release of calcium ions from intercellular storage room and the fact that calcium could stimulate NOX2 [42], we speculate that calcium may participate in RyR2-induced regulation of NOX2 in SCI. Further studies are needed to testify that hypothesis. Overall, we suggest that RyR2 play an important role in SCI through NOX2-mediated regulation of redox state and thus mitochondrial and ER dysfunction and inflammation (Fig. 6). The data provide novel insights into the mechanism of RyR2-mediated injury and the potential therapeutic targets for injury in spinal cord.

\section{Acknowledgments}

This work was supported by National Natural Science Foundation of China (No. 81372045).

\section{Disclosure Statement}

The authors declare that there are no conflicts of interest. 


\section{Cellular Physiology Cell Physiol Biochem 2016;38:1129-1137

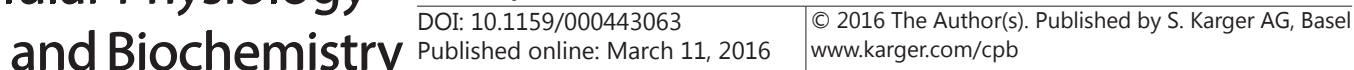

Liao et al.: Critical Role of Ryanodine Receptor 2 in Spinal Cord Injury

\section{References}

1 Wyndaele M, Wyndaele JJ: Incidence, prevalence and epidemiology of spinal cord injury: What learns a worldwide literature survey? Spinal Cord 2006;44:523-529.

2 Kim JH, Ahn SH, Cho YW, Kwak SG, Kim HS: Short-Term effect of percutaneous bipolar continuous radiofrequency on sacral nerves in patients treated for neurogenic detrusor overactivity after spinal cord injury: A randomized controlled feasibility study. Ann Rehabil Med 2015;39:718-725.

3 Moghimian M, Kashani F, Cheraghi MA, Mohammadnejad E: Quality of life and related factors among people with spinal cord injuries in tehran, iran. Arch Trauma Res 2015;4:e19280.

4 Bombardier CH, Adams LM, Fann JR, Hoffman JM: Depression trajectories during the first year after spinal cord injury. Arch Phys Med Rehabil 2016;97:196-203.

5 Hossain MS, Rahman MA, Bowden JL, Quadir MM, Herbert RD, Harvey LA: Psychological and socioeconomic status, complications and quality of life in people with spinal cord injuries after discharge from hospital in Bangladesh: A cohort study. Spinal Cord 2015; doi: 10.1038/sc.2015.179.

6 Block ML, Zecca L, Hong JS: Microglia-mediated neurotoxicity: Uncovering the molecular mechanisms. Nat Rev Neurosci 2007;8:57-69.

7 Fleming JC, Norenberg MD, Ramsay DA, Dekaban GA, Marcillo AE, Saenz AD, Pasquale-Styles M, Dietrich WD, Weaver LC: The cellular inflammatory response in human spinal cords after injury. Brain 2006;129:3249-3269.

8 Yamazaki D, Yamazaki T, Takeshima H: New molecular components supporting ryanodine receptormediated $\mathrm{Ca}(2+)$ release: Roles of junctophilin and TRIC channel in embryonic cardiomyocytes. Pharmacol Ther 2009;121:265-272.

9 Yin CC, D'Cruz LG, Lai FA: Ryanodine receptor arrays: Not just a pretty pattern? Trends in Cell Biology 2008;18:149-156.

10 Carrasco MA, Jaimovich E, Kemmerling U, Hidalgo C: Signal transduction and gene expression regulated by calcium release from internal stores in excitable cells. Biolog Res 2004;37:701-712.

11 Hamilton SL: Ryanodine receptors. Cell Calcium 2005;38:253-260.

12 Fabiato A: Calcium-induced release of calcium from the cardiac sarcoplasmic reticulum. Am J Physiol 1983;245:C1-C14.

13 Thorell WE, Leibrock LG, Agrawal SK: Role of RyRs and IP3 receptors after traumatic injury to spinal cord white matter. J Neurotrauma 2002;19:335-342.

14 Kesherwani V, Agrawal SK: Upregulation of RyR2 in hypoxic/reperfusion injury. J Neurotrauma 2012;29:1255-1265.

15 Chen MH, Liu YH, Xu H, Xu DW, Wang CN, Wang Y, Duan CW, Zhou Y, Kan P, Shen AG, Wang YH: Lentiviral Vector-Mediated p27 expression facilitates recovery after spinal cord injury. Molecular Neurobiology 2015; dx.doi.org/10.1007/s12035-015-9498-2.

16 Gruner JA: A monitored contusion model of spinal cord injury in the rat. J Neurotrauma 1992;9:123-126, 126-128.

17 Yang L, Ge Y, Tang J, Yuan J, Ge D, Chen H, Zhang H, Cao X: Schwann cells transplantation improves locomotor recovery in rat models with spinal cord injury: A systematic review and Meta-Analysis. Cell Physiol Biochem 2015;37:2171-2182.

18 Fang B, Li XQ, Bi B, Tan WF, Liu G, Zhang Y, Ma H: Dexmedetomidine attenuates blood-spinal cord barrier disruption induced by spinal cord ischemia reperfusion injury in rats. Cell Physiol Biochem 2015;36:373383.

19 Basso DM, Beattie MS, Bresnahan JC: A sensitive and reliable locomotor rating scale for open field testing in rats. J Neurotrauma 1995;12:1-21.

20 Wu J, Stoica BA, Dinizo M, Pajoohesh-Ganji A, Piao C, Faden AI: Delayed cell cycle pathway modulation facilitates recovery after spinal cord injury. Cell Cycle 2012;11:1782-1795.

21 Byrnes KR, Stoica BA, Fricke S, Di Giovanni S, Faden AI: Cell cycle activation contributes to post-mitotic cell death and secondary damage after spinal cord injury. Brain 2007;130:2977-2992.

22 Di Giovanni S, Knoblach SM, Brandoli C, Aden SA, Hoffman EP, Faden AI: Gene profiling in spinal cord injury shows role of cell cycle in neuronal death. Annals Neurol 2003;53:454-468. 


\section{Cellular Physiology Cell Physiol Biochem 2016;38:1129-1137

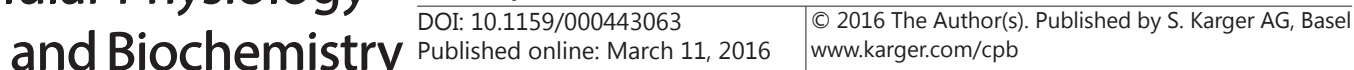

Liao et al.: Critical Role of Ryanodine Receptor 2 in Spinal Cord Injury

23 DePaul MA, Palmer M, Lang BT, Cutrone R, Tran AP, Madalena KM, Bogaerts A, Hamilton JA, Deans RJ, Mays RW, Busch SA, Silver J: Intravenous multipotent adult progenitor cell treatment decreases inflammation leading to functional recovery following spinal cord injury. Sci Rep 2015;5:16795.

24 Zhang B, Bailey WM, Kopper TJ, Orr MB, Feola DJ, Gensel JC: Azithromycin drives alternative macrophage activation and improves recovery and tissue sparing in contusion spinal cord injury. J Neuroinflamm 2015;12:218.

25 Miller DM, Singh IN, Wang JA, Hall ED: Nrf2-ARE activator carnosic acid decreases mitochondrial dysfunction, oxidative damage and neuronal cytoskeletal degradation following traumatic brain injury in mice. Expe Neurol 2015;264:103-110.

26 Wang Y, Wang J, Yang H, Zhou J, Feng X, Wang H, Tao Y: Necrostatin-1 mitigates mitochondrial dysfunction post-spinal cord injury. Neurosci 2015;289:224-232.

27 Li G, Jia Z, Cao Y, Wang Y, Li H, Zhang Z, Bi J, Lv G, Fan Z: Mitochondrial division inhibitor 1 ameliorates mitochondrial injury, apoptosis, and motor dysfunction after acute spinal cord injury in rats. Neurochem Res 2015;40:1379-1392.

28 Liu J, Du L: PERK pathway is involved in oxygen-glucose-serum deprivation-induced NF-kB activation via ROS generation in spinal cord astrocytes. Biochem Biophys Res Commun 2015;467:197-203.

29 Fan H, Tang HB, Kang J, Shan L, Song H, Zhu K, Wang J, Ju G, Wang YZ: Involvement of endoplasmic reticulum stress in the necroptosis of microglia/macrophages after spinal cord injury. Neuroscience 2015;311:362-373.

30 Zhang E, Yi MH, Shin N, Baek H, Kim S, Kim E, Kwon K, Lee S, Kim HW, Chul BY, Kim Y, Kwon OY, Lee WH, Kim DW: Endoplasmic reticulum stress impairment in the spinal dorsal horn of a neuropathic pain model. Sci Rep 2015;5:11555.

31 Luo Y, Fu C, Wang Z, Zhang Z, Wang H, Liu Y: Mangiferin attenuates contusive spinal cord injury in rats through the regulation of oxidative stress, inflammation and the Bcl2 and Bax pathway. Mole Med Rep2015;12:7132-7138.

32 Shou-Shi W, Ting-Ting S, Ji-Shun N, Hai-Chen C: Preclinical efficacy of Dexmedetomidine on spinal cord injury provoked oxidative renal damage. Ren Fail 2015;37:1190-1197.

33 Hart JE, Morse L, Tun CG, Brown R, Garshick E: Cross-sectional associations of pulmonary function with systemic inflammation and oxidative stress in individuals with chronic spinal cord injury. J Spin Cord Medicine 2015; dx.doi.org/10.1179/2045772315Y.0000000045.

34 Jiang ZS, Pu ZC, Hao ZH: Carvacrol protects against spinal cord injury in rats via suppressing oxidative stress and the endothelial nitric oxide synthase pathway. Mol Med Rep 2015;12:5349-5354.

35 Santulli G, Pagano G, Sardu C, Xie W, Reiken S, D'Ascia SL, Cannone M, Marziliano N, Trimarco B, Guise TA, Lacampagne A, Marks AR: Calcium release channel RyR2 regulates insulin release and glucose homeostasis. J Clin Investi 2015;125:1968-1978.

36 Li Q Su D, O'Rourke B, Pogwizd SM, Zhou L: Mitochondria-derived ROS bursts disturb Ca(2)(+) cycling and induce abnormal automaticity in guinea pig cardiomyocytes: A theoretical study. Am J Physiol Heart Circ Physiol 2015;308:H623-H636.

37 Wang H, Dong Y, Zhang J, Xu Z, Wang G, Swain CA, Zhang Y, Xie Z: Isoflurane induces endoplasmic reticulum stress and caspase activation through ryanodine receptors. Br J Anaesth 2014;113:695-707.

38 Liang J, Wu SY, Zhang D, Wang L, Leung KK, Leung PS: NADPH Oxidase-Dependent reactive oxygen species stimulate beta-Cell regeneration through differentiation of endocrine progenitors in murine pancreas. Antioxid Redox Signal 2015; dx.doi.org/10.1089/ars.2014.6135.

39 Rozycki M, Folke BJ, Speight P, Dan Q, Knudsen TE, Szeto SG, Yuen DA, Szaszi K, Pedersen SF, Kapus A: Myocardin-related transcription factor regulates Nox4 expression: Linking cytoskeletal organization to redox state. J Biol Chem 2015; 291:227-243.

40 Yang J, Qiu B, Li X, Zhang H, Liu W: P53-p66(shc)/miR-21-Sod2 signaling is critical for the inhibitory effect of betulinic acid on hepatocellular carcinoma. Toxicol Lett 2015;238:1-10.

41 Khayrullina G, Bermudez S, Byrnes KR: Inhibition of NOX2 reduces locomotor impairment, inflammation, and oxidative stress after spinal cord injury. J Neuroinflamation 2015;12:172.

42 Chen XH, Zhou X, Yang XY, Zhou ZB, Lu DH, Tang Y, Ling ZM, Zhou LH, Feng X: Propofol protects against HO -Induced oxidative injury in differentiated PC12 cells via inhibition of Ca-Dependent NADPH oxidase. Cell Mol Neurobiol 2015; doi.org/10.1007/s10571-015-0235-1. 